\title{
ARTIGO
}

\section{Investigación de fuentes de información jurídica en materia de discriminación}

\author{
Research into legal information \\ sources concerning discrimination
}

Alicia S. CAP ${ }^{1}$

\section{RE S U M E N}

El objetivo del proyecto fue elaborar estrategias de búsqueda eficaces, con el objeto de seleccionar citas para la composición de una bibliografía referente a la discriminación en el ámbito laboral a personas afectadas de SIDA. Se analizaron los términos de indización utilizados y la estructura de las fuentes de información, tanto impresas cuanto electrónicas, de uso más frecuente en el ámbito jurídico de la Argentina. Mediante el estudio comparativo del tratamiento que cada fuente da al tema, respecto a estructuración y términos de indización, se llegó a una hipótesis de trabajo para efectuar búsquedas específicas en materia de doctrina, legislación y jurisprudencia. En conclusión, se comprueba la efectividad de analizar la nomenclatura jurídica de los diferentes repertorios, con el fin de ajustar las estrategias de búsqueda a dichos términos y obtenerse el mayor grado de pertinencia de la información requerida, en el menor tiempo de recuperación.

Palabras claves: fuentes de información, nomenclatura jurídica, indización, SIDA discriminación laboral, doctrina, jurisprudencia, legislación, bibliografía, Argentina.

\footnotetext{
${ }^{1}$ Graduada de la Carrera de Bibliotecario Documentalista de la Facultad de Humanidades y Ciencias de la Educación, Universidad Nacional de La Plata. Estudiante de la Licenciatura y el Profesorado en la misma disciplina. Adscripta a la Cátedra de Catalogación I del Departamento de Bibliotecología. Colaboradora en carácter de auxiliar en Proyecto de investigación sobre "Control de autoridades en catálogos en línea", dirigido por la Prof. Ana M aría Martínez, Profesora Titular de Clasificación I.

Recebido e aceito para publicação em 8/7/2003.
} 


\section{A B S T R A C T}

This project's objective was to elaborate effective searching strategies, so as to select adequate citations to form a bibliography on discrimination of AIDSaffected people in the workplace. This paper presents the results of an analysis of the indexation terms and the structure of the information sources, both, printed and electronic, mostly used within the legal system in Argentina. The process involved a comparative analysis of each source's treatment of the referred subject, regarding structure and indexation terms. The results allowed some working hypotheses that led to specific searches regarding doctrine, legislation and jurisprudence. The effectiveness of analyzing the legal nomenclature of the different repertories, in order to adjust the searching strategies to such terms, was confirmed by the study's results. It made possible to retrieve, in the shortest amount of time, the most pertinent information on the required subject.

Key words: AIDS, indexation, information sources, legal nomenclature, discrimination in the workplace, doctrine, jurisprudence, legislation, Argentina.

\section{N T RO D U C C I Ó N}

En vista de la complejidad que las fuentes jurídicas presentan ante el requerimiento de información específica sobre un tema determinado, es fundamental el conocimiento de la estructura que cada una de ellas ofrece para poder elaborar estrategias de búsqueda eficaces, cuyos principales objetivos estarán encauzados a obtener el mayor grado de pertinencia de la información solicitada, en el menor tiempo de recuperación posible, se trate tanto de fuentes en soporte impreso como electrónico.

Dicha complejidad está dada por características particulares que presenta la información jurídica, a saber:

- Información de tipo acumulativo, en continuo crecimiento.

- Diversidad de la tipología de la documentación que la contiene: documentos primarios, secundarios y terciarios, cada uno de los cuales presenta en sí mismo una estructura particular y, a su vez, una estructura relacional entre ellos.

- Carácter interdisciplinario de la información: Esta característica de la información se refleja en la documentación jurídica que la contiene, orientada al logro de los siguientes fines:

- Transmitir nuevos conocimientos de naturaleza científica (Doctrina)

- Constituirse en elementos probatorios de los actos de la Administración Pública (Jurisprudencia)

- Reflejar el accionar de carácter oficial (Legislación).

La información de estos distintos tipos de documentación jurídica no se comporta en forma independiente una de otra sino que, por el contrario, se encuentra estrechamente relacionada entre sí. Así por ejemplo la jurisprudencia no puede prescindir para su elaboración ni de la doctrina ni de la legislación ya que ambas constituyen las bases primordiales de su fundamentación. Por su lado la doctrina no sólo cumple la función de sustentar a la Jurisprudencia, sino que por su carácter analítico e interpretativo sobre el sentido y razones de la legislación vigente, actúa también como fuente generadora de futuras normas, sobre aspectos jurídicos vacíos de estos contenidos.

- Especificidad de la información jurídica: no obstante su carácter relacional e interdisciplinario, cada temática del ámbito 
jurídico es unívoca y específica de un caso en particular.

- Carácter público de la información jurídica: el acceso a este tipo de información no es privativo de usuarios específicos del área jurídica ya que constituye un derecho extensivo a la sociedad en su conjunto.

Estos enunciados constituyen la base conceptual tomada como punto de partida para el desarrollo de esta investigación de Fuentes Jurídicas en materia de Discriminación. Los objetivos que persigue, junto con el alcance del tema, marco legal y estructura del producto final logrado, serán tratados a continuación.

El propósito final del presente trabajo es la elaboración de una Bibliografía que reúna en forma ordenada citas de los distintos tipos de documentos jurídicos en materia de discriminación.

Por motivos lógicos, el tema así planteado en forma tan general excedería los límites propuestos, tanto desde el punto de vista de la extensión del trabajo como de una optimización en la organización de la documentación obtenida, por lo cual se buscó ajustarlo, a partir de la generalidad, a una definición y delimitación lo más precisa posible, reduciéndolo a uno de sus aspectos.

Para el logro del objetivo final se emplearon varias estrategias las cuales no serán objeto de un análisis exhaustivo sino de una breve descripción que sea útil para que el lector pueda interpretar el criterio adoptado en el desarrollo y concreción del producto final de este trabajo, a saber:

- Conocer la estructura de las fuentes de información jurídica en soporte impreso y electrónico, es decir, la forma en que se encuentra organizada la información jurídica en materia de discriminación en cada una de ellas y las relaciones establecidas entre los distintos tipos de documentos que contienen (leyes, fallos, doctrina). El medio para lograr este objetivo es el análisis comparativo de la efectividad en la organización de los siguientes elementos: Fuentes impresas: Nomenclatura jurídica; Remisiones (por ejemplo, de una voz a otra); Puntos de acceso; Indices; Referencias contenidas en los distintos documentos jurídicos. Fuentes electrónicas: Puntos de acceso; Términos de indización; Grado de actualización de la información; Tipo de información (referencial, resúmenes, a texto completo).

- Definir a partir de este análisis las diferencias existentes entre las distintas fuentes y cuáles son las que presentan un mayor grado de pertinencia de los términos jurídicos y una organización clara de la información, que tienda a simplificar el acceso a ella en forma dinámica. Los medios para alcanzar este fin son la experimentación a través de búsquedas sobre el tema, partiendo de los términos jurídicos (o "voces") específicos que lo identifican y la observación de los resultados de las mismas en cuanto a pertinencia y tiempo efectivo de recuperación ya que estos factores son fundamentales en el momento en que se está frente a la exigencia de un usuario.

- Seleccionar los documentos jurídicos en materia de legislación, jurisprudencia y doctrina que se ajusten al alcance que se ha pretendido dar al tema de discriminación, de aquellas fuentes consideradas más efectivas y relevantes como producto de las conclusiones extraídas del análisis, experimentación y observación de los resultados obtenidos de las búsquedas efectuadas.

- Confeccionar las citas bibliográficas correspondientes a los documentos seleccionados que conformarán la bibliografía, otorgándoles un ordenamiento de tipo jerárquico.

Cabe aclarar que esta bibliografía no tendrá un carácter exhaustivo respecto al tema escogido sino que simplemente constituirá una reducida muestra del resultado obtenido como producto del desarrollo de la investigación realizada. La variedad y complejidad de las 
fuentes jurídicas requieren para su conocimiento en profundidad de una práctica intensiva, que no sólo consiste en comprender la estructura de las mismas sino también en adquirir cierta familiarización con la terminología jurídica, lo que no es posible lograr en breve lapso por lo cual, el objetivo de conocer dichas fuentes se ha fijado sólo como medio para llegar al propósito final.

\section{Alcance del tema}

El tema general escogido para el desarrollo de esta investigación es la discriminación en la República Argentina.

Presentada así, esta disciplina tiene dentro del marco legal de nuestro país una gama de manifestaciones muy amplia -raza, religión, nacionalidad, sexo, edad, opinión política o gremial, ideología, condición social, posición económica, caracteres físicos- y otras tantas que no son más que derivaciones y/o combinaciones de las mencionadas anteriormente pero que no están enunciadas en forma precisa dentro de la legislación vigente sobre discriminación, por lo cual se debe buscar la normativa correspondiente dentro de la cual se encuadran.

Por el carácter amplio que presenta el tema y por la inexistencia de una legislación taxativa referente a sus distintos aspectos, fue necesario delimitar su alcance a un campo bien definido para lo cual se partió de un análisis del concepto de discriminación, como así también del tratamiento normativo existente al respecto, con el fin de entrar en contexto.

En este sentido, desde un punto de vista socio-jurídico, la discriminación es considerada como el producto resultante de los modos en que los individuos que integran la sociedad interactúan, dentro de un marco de referencia constituido por el conjunto de normas sociales y jurídicas que rigen sus acciones, promoviéndolas hacia conductas positivas o negativas (FUCITO, 1993).
Las normas sociales (o informales) no se establecen legalmente sino que devienen del sistema cultural de la sociedad (usos, hábitos, costumbres, tradiciones), compartidas y respetadas por los miembros que la integran. En otros términos, son reglas generales comunes a la mayoría, transmitidas de generación en generación.

Las normas jurídicas (o formales) son consideradas como hechos sociales (FUCITO, 1993) entendidos dentro del contexto normativo de una cultura, en el que se le atribuyen significado y se le establecen las funciones que cumplen. A diferencia de las normas sociales, son creadas, promulgadas, sancionadas, interpretadas y aplicadas por organizaciones de la Administración Pública que actúan en función de intereses y necesidades condicionadas por el momento histórico-político reinante y las circunstancias de tiempo y de lugar (GERLERO, 1995).

Esto confiere a las normas jurídicas un carácter mutable y, por ende, temporal y la posibilidad de convertirse tanto en instrumento de control social del Estado como en agente de cambio social, lo que estará íntimamente vinculado a la estrategia y forma en que la Administración Pública emplee la normativa jurídica para resolver los fenómenos sociales problemáticos (GERLERO, 1995).

Pero es necesario señalar que, por la naturaleza dinámica que caracteriza a toda sociedad, los componentes que la conforman no se comportan de manera inflexible dentro de ese marco normativo social y jurídico descripto, como si fuera tan solo un esquema. Ella constituye un sistema en el que coexisten numerosos subsistemas algunos de los cuales están representados por agrupamientos sociales o individuos con diferentes pautas culturales y de comportamiento respecto a la generalidad, que definen modelos propios de conducta, los cuales pueden contraponerse parcial o totalmente al sistema de normas comunes al resto de la 
sociedad y que, si bien no influyen sobre los demás, establecen una diferencia que genera rechazo y marginación de estos últimos, los demás, hacia dichos individuos o agrupamientos sociales y que se traduce en actos discriminatorios.

El Derecho cumple un rol esencial en el tratamiento de los actos discriminatorios ya que constituye el conjunto de normas sancionadas por el Estado, según un procedimiento determinado y considerado válido en un territorio (GERLERO, 1995).

De acuerdo a la estrategia que la Administración Pública siga a partir de la normativa jurídica vigente, ésta resultará efectiva si trata de armonizar y compatibilizar los distintos intereses y necesidades sociales hacia conductas no discriminatorias (positivas), solucionando de esta manera fenómenos sociales problemáticos (GERLERO, 1995).

No obstante puede ocurrir también que la normativa jurídica empleada por la Administración Pública no sea efectiva, provocando un agravamiento del fenómeno social problemático y generando ella misma conductas discriminatorias (negativas) (GERLERO, 1995).

No es objeto de este trabajo tomar como referentes ambos casos sino sólo aquel en el que la Administración Pública utiliza dicha normativa como instrumento no discriminatorio, haciendo respetar el derecho a ser "diferente" que poseen aquellas personas o grupos sociales cuyo comportamiento no se identifica con las reglas generales de la mayoría, sin que ello implique una influencia sobre ella.

Ejemplos de estos grupos sociales "diferentes"en la actualidad lo constituyen, entre otros, el de los homosexuales y, muy vinculados con ellos y otros grupos minoritarios, aquellos que son estigmatizados, perseguidos y discriminados por padecer SIDA.

Indagando dentro de cada una de las manifestaciones en que la discriminación se presenta dentro de la sociedad, los actos discriminatorios hacia los enfermos de SIDA despierta un particular interés en primer lugar porque la enfermedad ha dejado de circunscribirse a los grupos homosexuales, registrándose un marcado incremento de víctimas dentro de la comunidad heterosexual, o sea en personas que no necesariamente tienen conductas o normas sociales diferentes a las de la mayoría pero que, sin embargo, por el solo hecho de padecer SIDA pasan a constituir un nuevo grupo marginado y rechazado por los demás.

Por otro lado, si bien no existen pruebas de transmisión del VIH mediante otras formas de contacto que las conocidas (relaciones sexuales, productos sanguíneos infectados, material contaminado, de madre a hijo durante el embarazo o después del parto), el impacto psicológico, social y económico sobre la persona infectada y su entorno inmediato, como así también en la sociedad, es de una gran envergadura, generando lo que la Organización Mundial de la Salud (OMS) ${ }^{2}$ ha dado en llamar la tercera epidemia, caracterizada por la persecución y discriminación de los infectados por el VIH en todos los ámbitos en los que se desenvuelve su vida de relación (laboral, cultural, social).

Estas conductas discriminatorias originadas como reacción de defensa de la sociedad ante la epidemia del SIDA y cuya consecuencia directa es evitar el contacto con personas infectadas por el VIH generando su segregación, no responden a prejuicios sociales como en los casos referentes a raza, religión, sexo, ideología, etc. sino que son en definitiva

\footnotetext{
2 Según la OMS existen tres epidemias que representan, cada una, fases de la invasión de la comunidad por el virus del SIDA. La primera es la epidemia del contagio silencioso, a menudo desconocido; la segunda después de un plazo de varios años, es la epidemia de la enfermedad del SIDA y la tercera, la descripta precedentemente (COMISIÓ N..., 1988 p. 38)
} 
producto de un profundo desconocimiento relativo a la génesis, características y peculiaridades de la enfermedad, potenciado por el temor al contagio, y afectan un derecho personalísimo básico de todo individuo: la igualdad.

Al avanzar en la investigación sobre este aspecto específico de la discriminación, se descubre que la segregación y el despojo del derecho a la dignidad humana en los enfermos de SIDA encuentran su manifestación más despiadada dentro del ámbito laboral al que pertenecen, a través de despidos injustificados a los que son sometidos, una vez confirmada la infección por el virus o la condición de ser portadores sanos del VIH.

Los conflictos generados por la segregación laboral de los seropositivos son una muestra cabal de cómo reacciona la comunidad frente al SIDA que se ha convertido, no solo en una fuente de marginación social, sino en un medio de destrucción de la coexistencia de los distintos grupos que conforman la sociedad (POSE, 1997b).

En verdad, los enfermos de SIDA son considerados en la actualidad un "grupo de riesgo laboral" y se los discrimina porque se teme su presencia como fuente de contagio en la empresa, razón por la cual se los desplaza de su puesto de trabajo y/o se rechaza toda posibilidad de contratación de su persona (POSE, 1997b). Esto refleja una fuente de perjuicio mucho más grave que la existente respecto a las conductas discriminatorias por razones de raza, sexo, etc., porque los despidos se producen en forma sutil sin invocar una causa o invocando otra distinta a la que realmente originó la medida, que se oculta por ser discriminatoria.

Pero el riesgo de discriminación en materia de empleo en el caso de los enfermos de SIDA encierra en sí mismo un doble aspecto negativo, ya que se produce en los dos extremos de la relación laboral por cuanto, como ya se dijo, es tan difícil para los seropositivos obtener empleo, como fácil que se prescinda de sus servicios por razones injustificadas.

De todo lo expuesto hasta aquí se concluye que la Discriminación laboral en personas afectadas de SIDA, como así también en portadores sanos del VIH, afecta varios derechos fundamentales de toda persona tales como el derecho al trabajo, a la no discriminación y a la salud, cuya manifestación más evidente es el despido injustificado y algunas otras formas que éste adopta en determinadas circunstancias, como el retiro obligatorio de trabajadores afectados por la enfermedad o sospechados de estarlo.

La dificultad en la búsqueda de información sobre el tema de discriminación dentro del marco de referencia señalado precedentemente, discriminación laboral vinculada a discriminación por enfermedad, exige un desafío interesante ya que se debe indagar dentro de ambos campos, como así también dentro de su manifestación directa, el despido injustificado.

El mencionado desafío está basado fundamentalmente en la elaboración de estrategias de búsqueda correctas a partir de la utilización de términos jurídicos pertinentes a los principios que son afectados por estas conductas discriminatorias (igualdad ante la ley, garantías y derechos constitucionales, etc.) y a sus manifestaciones (por ejemplo, daños y perjuicios, daño moral, despido, etc.), ya que no es posible acceder a la información sobre el tema a través de la palabra discriminación ni de ninguna de sus variantes (discriminación laboral, discriminación por enfermedad, etc.) porque no forman parte de la nomenclatura jurídica, a lo que se debe sumar la falta de uniformidad de esta última en los distintos tipos de fuentes.

Estos aspectos, a los que se agregan el interés particular en investigar el tratamiento que el Derecho ha dado al tema de Discriminación laboral en personas afectadas de SIDA, han sido los motivos determinantes de la definición y 
delimitación del alcance del tema general de discriminación dentro de ese marco de referencia.

Para introducir el tema de Discriminación laboral en personas afectadas de SIDA dentro del marco legal que le corresponde es preciso previamente establecer el alcance temporal y geográfico que ha de tener el mismo ya que toda normativa requiere de una ubicación en tiempo y espacio para que cobre vida.

En cuanto al alcance temporal es importante señalar que, si bien se desconoce con exactitud la fecha de inicio del SIDA, lo cierto es que la epidemia irrumpe mundialmente en los primeros años de la década de 1980, propagándose solapadamente desde América del Norte (donde se descubren los primeros casos entre homosexuales), a través de fronteras nacionales, étnicas, culturales y sexuales (COMISIÓN INTERNACIONAL DE JURISTAS..., 1988).

De acuerdo con este referente y tomando en cuenta las consideraciones de la OMS relativas a las fases de la epidemia, expuestas precedentemente en la página 4, se elaboró la hipótesis de la imposibilidad de ubicar jurisprudencia en los cinco años posteriores a 1980 sobre la base del supuesto de que en dicho lapso la enfermedad se encontraba en pleno proceso de "contagio o desarrollo silencioso" en las personas que la habían adquirido, por lo cual no sentó jurisprudencia en dicho período.

A partir de estas premisas se comenzó desde 1985 a investigar haciendo búsquedas sobre el tema sin resultados positivos. Recién a partir de los repertorios de 1993 en adelante se logró localizar información sobre el tema del SIDA, por lo cual el desarrollo del trabajo está prácticamente comprendido en el período 1993-2001.

El alcance geográfico se ha restringido casi exclusivamente al ámbito nacional, ya que a medida que se iba experimentando con las búsquedas, la información hallada respondía en su mayoría a esa jurisdicción, que de todas formas no es muy abundante como se podrá apreciar en la Bibliografía, sobre todo en lo relativo a jurisprudencia.

No obstante, se elaboraron estrategias para efectuar lo propio dentro del ámbito provincial, más específicamente en la Provincia de Buenos Aires, con resultados más pobres aún dado que se pudieron localizar sólo dos fallos, que se incluyeron dentro de la Bibliografía junto con la legislación provincial vigente en materia de discriminación.

\section{Marco Legal}

Una vez definido y delimitado el alcance del tema general y de haber establecido su ubicación en tiempo y espacio, fue preciso introducir el tema específico resultante Discriminación laboral en personas afectadas de SIDA- dentro del marco legal que le corresponde. Dicho en otros términos, habiendo decidido qué es lo que se quería indagar del tema de discriminación, el punto de partida para comenzar las búsquedas de la información respectiva, fue analizar la normativa vigente en la que se encuadra.

Como se mencionó precedentemente el SIDA, analizado dentro del contexto de las relaciones laborales, pone en juego varios derechos fundamentales tales como el derecho al trabajo, a la no discriminación y a la salud.

Sin embargo, la discriminación que genera el SIDA está tratado dentro de nuestra legislación nacional de manera muy poco precisa.

De hecho, casi no existen normas laborales que expresamente se refieran a la Discriminación laboral en personas afectadas de SIDA y regulen, por ejemplo, los despidos arbitrarios. En este sentido tampoco hay normas internacionales relativas al trabajo, que se refieran específicamente a este tema. Sólo se cuenta con una serie de normas constitucionales y tratados internacionales con jerarquía 
constitucional, cuyos enunciados son principios generales de aplicación frente a los fenómenos problemáticos originados por el SIDA en el contexto de las relaciones laborales.

Por ejemplo, en la Constitución Nacional (CONSTITUCIÓN DE LA NACIÓN ARGENTINA..., 2001) se encuentran una serie de normas cuyos enunciados condenan las conductas discriminatorias, ya sea en forma tácita o manifiesta, abasteciendo importantes instrumentos legales. Ellas son:

Artículo 16. En el que se pone de manifiesto en forma tácita la prohibición de conductas discriminatorias a través de la consagración de la regla de igualdad.

Artículo 43. En este artículo se consagra el reconocimiento a interponer acción de amparo contra cualquier forma de discriminación.

Artículo 75 inc. 22. En este inciso se dispone la incorporación a la Constitución Nacional (reforma de 1994) de numerosos Convenios y Pactos Internacionales con jerarquía superior a las leyes, los cuales dan cuenta de la igualdad de trato y la prohibición de discriminación de cualquier índole.

Artículo 75 inc. 23. Se establece en este artículo que le corresponde al Congreso de la Nación: legislar y promover medidas de acción positivas que garanticen la igualdad real de oportunidades y de trato, y el pleno goce del ejercicio real de los derechos reconocidos por la Constitución y los Tratados Internacionales vigentes sobre derechos humanos.

Respecto a la legislación nacional vigente en materia de discriminación nuestro país cuenta con la Ley 23.592, conocida como Ley de la Rúa o Ley Antidiscriminatoria, que considera como factores particularmente discriminatorios la raza, la religión, la nacionalidad, la ideología, la opinión pública y gremial, el sexo, la posición económica, la condición social y/o los caracteres físicos. Si bien no se incluye dentro de los mencionados factores la discriminación por motivos de salud, es una norma enunciativa tomada en cuenta en el tratamiento del tema en cuestión.

También la Ley 20.744 de Contrato de Trabajo reviste este carácter al disponer en su artículo 17 la prohibición de cualquier tipo de discriminación entre los trabajadores por motivos de sexo, raza, nacionalidad, religiosos, políticos, gremiales o de edad, no previendo la discriminación por razones de salud.

El artículo 81 de esta misma ley establece la igualdad de trato que el empleador debe dispensar a los trabajadores en igualdad de condiciones, considerando que habrá trato desigual cuando se produzcan discriminaciones arbitrarias fundadas en razones de sexo, religión o raza. Este artículo tampoco contempla las discriminaciones por enfermedad.

Por último, la única norma vigente en materia de SIDA, la Ley 23.798 de Lucha contra el SIDA, declarada de interés nacional y cuyo objetivo prioritario es la educación de la población, veda respecto a las personas afectadas de SIDA, cualquier acto discriminatorio, aunque en este último sentido no lo hace en forma taxativa.

Por su parte el artículo 11 de la Constitución de la Provincia de Buenos Aires consagra la igualdad ante la ley de todos los habitantes de la Provincia estableciendo además que en ella no se admiten distinciones, discriminaciones ni privilegios por razones de sexo, raza, religión, nacionalidad, lengua, ideología, opinión, enfermedades de riesgo, características físicas o cualquier otra condición amparada por las normas constitucionales. $\mathrm{Si}$ bien se condenan en forma manifiesta las conductas discriminatorias por enfermedades de riesgo, no deja de ser meramente enunciativo el contenido de este artículo, pero no por ello irrelevante.

Para la definición del marco legal se consultaron las siguientes fuentes:

En soporte impreso: 
- Boletín oficial de la República Argentina: órgano de publicación de las leyes nacionales

- Constitución Nacional: se localizaron los artículos y tratados internacionales con fuerza de ley que dan tratamiento al tema de discriminación.

- Constitución Provincial: se buscaron en este documento los artículos correspondientes a discriminación que rigen dentro de la jurisdicción de la Provincia de Buenos Aires.

- Anales de legislación argentina / La Ley: repertorio en el que se ubicaron las leyes nacionales.

En soporte electrónico:

- Infoleg, en el que se indagaron algunas leyes, como por ejemplo la ley antidiscriminatoria en cuanto a relación (modificaciones) con otras leyes. No fue posible obtener el texto completo de la misma sino sólo un breve resumen de su contenido y un cuadro de las vinculaciones que presenta con otras normas.

Las fuentes consultadas en materia de jurisprudencia y doctrina fueron las siguientes:

En soporte impreso:

- Revista jurídica argentina La ley / La ley

- La ley Buenos Aires / La ley

- Jurisprudencia Argentina / Jurisprudencia Argentina Argentina

- El derecho / Universidad Católica

- Derecho del trabajo / La ley

- Trabajo y seguridad social / El derecho

En soporte electrónico:

- FANA en línea: Se consultó para la búsqueda de fallos, solo a título referencial ya que no hay documentos a texto completo en esta base de datos.

- JUBA en línea: También se consultó solo a título referencial ya que no hay documentos a texto completo en esta base de datos.
- SAIJ: Esta base de datos es muy efectiva y muy completa pero no se puede acceder en forma gratuita por lo cual no se pudo utilizar.

\section{E TO D O L O G ÍA}

El criterio adoptado para el establecimiento del marco legal que le corresponde al tema de discriminación por SIDA dentro del ámbito laboral, es producto de varias estrategias de búsqueda que debieron implementarse en forma sistemática dada la complejidad que el tema en cuestión adquiere al no ajustarse a una norma específica en particular, sino a varias de carácter enunciativo.

Se consideró prioritario comenzar por encuadrar el tema dentro del marco legal que le corresponde porque es el orden lógico para lograr aproximaciones a la documentación buscada a través de la utilización de diversas estrategias, para cuya elaboración fue necesario incursionar en la doctrina existente, tanto en materia de discriminación en su forma genérica, como en la referente a SIDA y a discriminación laboral, medio por el que se lograron precisar las voces que en el ámbito jurídico identifican el tema.

En esta línea conceptual es importante destacar, por ejemplo, en lo que respecta a fuentes impresas, que el término discriminación no se encontró como tal dentro de la nomenclatura jurídica, por lo cual se comenzaron las búsquedas por otras voces tales como igualdad ante la ley, derechos y garantías y/o derechos personalísimos. A medida que se profundizó en la investigación, encauzando las búsquedas hacia el campo más específico, se observó que por el carácter relacional de la información, una gama más amplia de voces, como por ejemplo actos ilícitos, daños y perjuicios, daño moral, despido indirecto, despido arbitrario, permite acceder al mismo, pero guardando en todo momento un vínculo con los principios o derechos que son afectados 0 
vulnerados (como por ejemplo los mencionados precedentemente), y no con su consecuencia última, la discriminación, dato importante a tener en cuenta para elaborar las estrategias.

Los términos de indización de la información jurídica sobre el tema en cuestión en fuentes electrónicas se comporta de manera diferente, ya que no está estructurada de la misma manera que en las fuentes impresas. Es factible iniciar búsquedas de una manera más dinámica a través del término discriminación, sida o de ecuaciones en las que se combinen estas voces con otras relativas al tema específico, agregando a esto las posibilidades de enlaces que podemos efectuar entre los distintos tipos de documentos, como así también entre diferentes sitios.

El inconveniente con muchos de estos tipos de fuentes, en especial los de acceso gratuito, es que no permiten acceder en todos los casos al texto completo del documento requerido, ofreciendo solo datos referenciales y/o un resumen de su contenido.

En relación al tema en cuestión no ha sido posible encontrar en materia de legislación ni de jurisprudencia, documentos a texto completo en fuentes electrónicas, sino solo resúmenes, por lo cual la bibliografía básicamente contiene citas tomadas de fuentes impresas.

\section{Estructura de la bibliografia}

La documentación que contiene la Bibliografía en materia de Discriminación laboral en personas afectadas de SIDA, está agrupada de acuerdo al rango jerárquico que le corresponde, esto es, legislación nacional en primer término, luego legislación provincial, continuando a posteriori con jurisprudencia para finalizar con doctrina. A continuación se describen algunos detalles de importancia que presenta la estructura de la Bibliografía, a saber:

\section{Encabezamientos y ordenamiento de las citas}

Tratados internacionales: Encabezamiento por título del tratado; ordenamiento por fecha de entrada en vigor del tratado.

Leyes nacionales: Encabezamiento por número de ley; ordenamiento cronológico por fecha de publicación de la ley en el Boletín oficial.

Jurisprudencia: Encabezamiento por demandante; ordenamiento alfabético.

Doctrina: Encabezamiento por autor / es; ordenamiento alfabético.

\section{Abreviaturas}

Las partes intervinientes en los fallos se describen en el caso de los demandantes con las iniciales de sus nombres y apellidos, mientras que el nombre de los demandados se presenta in extenso. Esto responde a lo establecido en la ley 23.798 respecto a la preservación de los datos de identificación del enfermo de SIDA. En algunos fallos los demandantes son mencionados con las siglas $\mathrm{S} / \mathrm{N}$, sin nombre.

Los datos de tribunal se citan en forma abreviada y a continuación la fecha del fallo.

Los repertorios en los que aparecen publicados los documentos citados fueron registrados en forma abreviada (Anexo).

Para conocer el nombre in extenso de cualquiera de estas abreviaturas se debe consultar la lista de abreviaturas que se presenta al comienzo de la Bibliografía.

\section{Remisiones}

Las notas a fallo remiten al fallo correspondiente por medio de referencias de véase además. El fallo a su vez indica que posee una nota a fallo mediante una nota de con y una 
referencia de véase además remite al autor y título de la nota a fallo correspondiente.

Los repertorios citados en cada registro bibliográfico contienen el documento a texto completo. En algunos casos se establecen remisiones a otros repertorios que contienen el mismo documento, a través de la indicación "Publicado también en":

\section{Puntuación}

Los artículos de la Constitución y de los Tratados Internacionales, están precedidos de dos puntos en aquellos casos en que estén contenidos en partes y/o capítulos. Estos últimos también estarán precedidos de este signo de puntuación cuando se encuentren dentro de una sección o parte.

La paginación está precedida de dos puntos y la indicación de volumen y/o número de la publicación en el caso de artículos de revistas, y de la abreviatura -p.- en el caso de citas de libros y/o partes contenidas en los mismos.

\section{O N CLUSIONES}

La Bibliografía resultante de esta investigación no es obra de una experiencia

\section{REFERE N C IAS}

ARGENTINA. Constitución de la Nación ARGENTINA. Buenos Aires: Zavalía, 2001. Cap. 1: art. 16. Cap. 2: art. 43. Cap. 4: art. 75, inc. 22 y 23, p. 12, 19, 28-29.

COMISIÓN INTERNACIONAL DE JURISTAS. Sida y discriminación. La Revista CIJ, Ginebra, v.41, p.3842, 1988. acabada sino simplemente una aproximación al comienzo del ejercicio de una práctica basada en la utilización de las fuentes jurídicas, sobre la base del conocimiento real acerca de la complejidad de las mismas.

Complejidad que obliga no sólo a descubrir la forma en que está estructurada y organizada la información, sino también a aplicar un criterio analítico de la terminología empleada en cada una de las fuentes, ya que estos dos aspectos constituyen la base esencial para lograr armar estrategias de búsqueda correctas que conduzcan de una manera dinámica a la obtención de resultados óptimos, ante los requerimientos de información sobre un tema específico.

Dichas estrategias siempre orientan hacia un resultado concreto que, aunque no sea el deseado, permite conocer nuevos puntos de acceso que sirven de base para su reelaboración o para su descarte definitivo.

En conclusión, el presente trabajo sólo constituye una reducida muestra de cómo se ha llegado a recopilar la información en materia de discriminación laboral en personas afectadas de SIDA, a través de la investigación de las fuentes jurídicas existentes, por lo cual el propósito final se considera parcialmente logrado ya que queda aún mucho por investigar para obtener el producto final deseado.

ERMIDA URIARTE, O. Sida y derecho laboral. In: TySS, Buenos Aires, v.3, p.273-279, 1994.

FUCITO, F. Sociología del derecho. Buenos Aires: Universidad, 1993. p.22-25.

GERLERO, M.S. Discriminación: una aproximación teórica desde la sociología del derecho. Buenos Aires: Iniciales, 1995. p.1-15. 
Ley 20.744. Ley de Contrato de Trabajo. Arts. 18 y 89. BO, Buenos Aires, Argentina, 27 de septiembre de 1974. Publicada también en: ADLA, 1974; XXXIV-D: 3245-3246.

Ley 23.592. BO. Buenos Aires, Argentina, 5 de septiembre de 1988. Publicada también en: ADLA, 1988; XLVIII-D: 4179-4180.

\section{B IB LIO G RAFIACO N S LTA DA}

\section{LEGISLACION NACIONAL / CONSTITUCIÓN NACIONAL}

TRATADOS INTERNACIONALES CON JERARQUIA CONSTITUCIONAL (Art. 75, inc. 22 de la Constitución de la Nación Argentina)

DECLARACIÓN americana de los derechos y deberes del hombre. Conferencia Internacional Americana (9a: 1948: Bogotá). Cap. 1: art. 2, p. 57. In: ARGENTINA. Constitución de la Nación Argentina. Buenos Aires: Zavalía, 2001. Ap. 1. Instrumentos internacionales con jerarquía constitucional.

DECLARACIÓN universal de derechos humanos. Adoptada y proclamada por la Asamblea General de las Naciones Unidas en su Resolución 217 A (III) del 10 de diciembre de 1948. Art. 2: pto. 1 y art. 7; p. 65-66. In: ARGENTINA. Constitución de la Nación Argentina. Buenos Aires: Zavalía, 2001. Ap. 1. Instrumentos internacionales con jerarquía constitucional.

PACTO internacional de derechos económicos, sociales y culturales. Adoptado y proclamado por la Asamblea General de las Naciones Unidas en su Resolución 2200 A (XXI) del 16 de diciembre de 1966, entrada en vigor el 3 de enero de 1976. Pte. 2: art. 2: pto. 2; p. 109. In: ARGENTINA. Constitución de la Nación Argentina. Buenos Aires: Zavalía, 2001. Ap. 1. Instrumentos internacionales con jerarquía constitucional.

PACTO internacional de derechos civiles y políticos. Adoptado por la Asamblea General de las Naciones Unidas en su Resolución 2200 A (XXI) del 16 de diciembre de 1966, entrada en vigor el 23 de marzo
Ley 23.798. Art. 2. BO. Buenos Aires, Argentina, 20 de septiembre de 1990. Publicada también en ADLA, 1990; L-D: 3627.

POSE, C. Un caso de despido por Sida. Nota a fallo. $D T$, Buenos Aires, v.57, n.3, p.496-499, 1997b.

de 1976. Pte. 2: art. 2: pto. 1 y pte. 3: art. 26, p. 125 y 136. In: ARGENTINA. Constitución de la Nación Argentina. Buenos Aires: Zavalía, 2001. Ap. 1. Instrumentos internacionales con jerarquía constitucional.

CONVENCIÓN americana sobre derechos humanos. Conferencia Especializada Interamericana sobre Derechos Humanos (1969: nov. 22: San José de Costa Rica), entrada en vigor el 18 de julio de 1978. Pte. 1: Cap. 1: art. 1, Cap. 2: art. 24, p. 74, 84. In: ARGENTINA Constitución de la Nación Argentina. Buenos Aires: Zavalía, 2001. Ap. 1. Instrumentos internacionales con jerarquía constitucional.

CONVENCIÓN sobre la eliminación de todas las formas de discriminación contra la mujer. Adoptada por la Asamblea General de las Naciones Unidas en su Resolución 34 / 180, del 18 de diciembre de 1979, entrada en vigor el 3 de septiembre de 1981. Pte. 1: arts. 1 y 2, p. 184-185. In: ARGENTINA Constitución de la Nación Argentina. Buenos Aires: Zavalía, 2001. Ap. 1. Instrumentos internacionales con jerarquía constitucional.

ARGENTINA. Lei n. 20.744, de 27 de septiembre de 1974.

ADLA, Buenos Aires, v. 34-D, p. 3245-3246, 1974.

\section{DECRETOS}

DECRETO $390^{*}$. Régimen de contrato de trabajo. Texto ordenado del aprobado por ley 20.744 y modificado por Ley 21.297. Arts. 17 y 81. BO. Buenos Aires, Argentina, 21 de mayo de 1976. Publicado también en ADLA, 1976; XXXVI-B : 1176, 1182.

${ }^{(*)}$ A través de este decreto los arts. 18 y 89 de la Ley 20.744 pasan a constituir los arts. 17 y 81, respectivamente. La Ley 21.297 no modifica el contenido de los mismos. 


\section{LEGISLACION PROVINCIAL / CONSTITUCIÓN PROVINCIAL}

ARgentinA. Constitución de la Provincia de Buenos Aires. [en línea] La Plata, 1994. Secc. 1a: arts. 11, 12: inc. 3, 20, 36. inc 4. Disponible en: <http://infoleg.mecon.gov.ar.> e <http://www.gob. gba.gov.ar>. Acesso en: 29 oct. 2001.

\section{JURISPRUDENCIA}

B., H. c Empresa Líneas Marítimas Argentinas. CNTrab., sala III, marzo 30-1995. JÁ, Buenos Aires, v. 4, p.102-104, 1995.

I.,O. E. c Empresa Líneas Marítimas Argentinas S. A. CNTrab., sala VII, mayo 31-1996. Con nota a fallo. DT, Buenos Aires, A. p.496-499, 1997.

véase además Pose, Carlos. Un caso de despido por Sida.

J., J. c Materia Hnos S. A. TTrab. No. 2, Mar del Plata, agosto 28-1997. TySS, Buenos Aires, v.11, p.1043-1045, 1997.

J., J. c Materia Hnos S. A. C1a.Civ. y Com., Mar del Plata, sala II, agosto 10-2000. Con nota a fallo. LLBA, Buenos Aires, v.8, n.6, p.758-765, 2001.

véase además Goldenberg, Isidoro $\mathrm{H}$. Discriminación laboral. Derechos personalísimos

M., J. O. c Cafés Chocolates Aguila y Productos Saint Hnos. S. A., CNTrab., sala X, diciembre 31-1997. DT, Buenos Aires: A, 1998. p.1237.

S/N c Policía Federal Argentina. CS, diciembre 17-1996. Con nota a fallo. In: ED. Buenos Aires: UCA, 1997. 172, p.329-342. Publicado también en: LL. Buenos Aires: LL, 1997; D: 253-263 // DT. Buenos Aires: LL, 1997; 57(9): 1779-1791.

véase además Andruet, Armando S. (h). Dignidad humana. Intimidad personal y Sida // Pose, Carlos. Protección laboral del sidótico a través de un pronunciamiento de la Corte Suprema de Justicia de la Nación.

T., P. c Editorial Sarmiento S. A. CNCiv., sala I, abril 3-1997. En: LL. Buenos Aires: LL, 1997. D, p. 398-409. Publicado también en: ED, 1997; 173: 120-123 // JA, 1998; I: 326-329 // TySS, 1997; 11: 1029-1043. véase además Vázquez Vialard, Antonio. Extinción del contrato de trabajo: El despido indirecto discriminatorio.

V., R. M. c Empresa Líneas Marítimas Argentinas S. A. CNTrab., sala V, septiembre 30-1993. Con nota a fallo. In: LL. Buenos Aires: LL, 1994. B: 233. Publicado también en: DT, 1994; 54(2): 2116-2121 // TySS, 1994; 3: 324-326.

véase además Poclava Lafuente, Juan C. Sida y trabajo.

\section{DOCTRINA}

ANDRUET, H.; ARMANDO, S. Dignidad humana: intimidad personal y Sida. Nota a fallo. ED. Buenos Aires: UCA, 1997. v.172, p.323-327.

véase además S/N c Policía Federal Argentina, CS, diciembre 17-1996

BECKER, H. Los extraños. In: Sociología de la desviación. Buenos Aires: Tiempo Contemporáneo, 1974. p.15.

GARCÍA, M.R. La igualdad de trato y la discriminación en el derecho del trabajo. DT, Buenos Aires, v.57, n.5, p.891-896, 1997.

GIDDENS, A. La constitución de la sociedad. Buenos Aires: Amorrortu, 1995. p.51.

GOLDENBERG, I.H. Discriminación laboral: derechos personalísimos. Nota a fallo. LLBA, Buenos Aires, v.8, n.6, p.758-761, 2001.

véase además J., J. c Materia Hnos. S.A., C1a.Civ. y Com., Mar del Plata, agosto 10-2000

GOLDENBERG, I.H.; PIZARRO, D.R. El derecho frente a la discriminación. In: JORNADAS NACIONALES DE DERECHO CIVIL, 15., 1995. Mar del Plata. Buenos Aires: JA, 1995. v.4, p.833-841. GONZÁLEZ (h), R.O. El sida es sinónimo de despido? Buenos Aires: LL, 1998. v.9, p.123.

GRMEK, M. Historia del sida. México: Siglo XXI, 1992.

Legislatura de la Ciudad Autónoma de Buenos Aires. Comisión de Derechos Humanos, Garantías y Antidiscriminación. Digesto de normas antidiscriminatorias. Buenos Aires: Legislatura de la Ciudad Autónoma de Buenos Aires, 2001. 
MARTÍNEZ V., J.J. La discriminación en el contexto actual de las relaciones laborales. DT, Buenos Aires, v.57, n.9, p.1731-1736, 1997.

MONTI, J.E. El sida y el hombre. Buenos Aires: Alcotan, 1993.

ORGANIZACIÓN Internacional del Trabajo. SIDA: Plan mundial y nuevo Repertorio de recomendaciones prácticas. Revista de la OIT, Ginebra, v.40, n.4-6, 2001.

POCLAVA LAFUENTE, J.C. Sida y trabajo. Nota a fallo. I: LL. Buenos Aires: LL, 1994. B: p.232.

véase además V., R. M. c Empresa Líneas Marítimas Argentinas S.A. CNTrab., sala V, septiembre 30-1993.

véase además I., O. E. c Empresas Líneas Marítimas Argentinas S.A., CNTrab., mayo 31-1996
POSE, C. Protección laboral del sidótico a través de un pronunciamiento de la Corte Suprema de Justicia de la Nación. DT, Buenos Aires, v.57, n.9, p.1779-1791, 1997a.

véase además S/N c Policía Federal Argentina, CS, diciembre 17-1996.

VAZ FLORES, H. Localización, transferencia y uso de la información jurídica. Revista del Colegio de Abogados de La Plata, La Plata, v.25, n.45, p.183.

VÁZQUEZ VIALARD, A. Extinción del contrato de trabajo: el despido indirecto discriminatorio. TySS, Buenos Aires, v.11, p.1046-1052, 1997.

véase además T., P. c Editorial Sarmiento S.A., CNCiv., abril 3-1997.

VES LOSADA, A. Sociología del derecho. Buenos Aires: Depalma, 1980.

\section{ANEXO}

\section{LISTA DE ABREVIATURAS PUBLICACIONES}

\begin{tabular}{ll}
\hline ADLA & Anales de legislación argentina / La ley \\
BO & Boletín Oficial de la República Argentina \\
DT & Derecho del trabajo / La ley \\
日D & El derecho / Universidad Católica Argentina \\
IMP & Impuestos / La ley \\
JA & Jurisprudencia argentina / Jurisprudencia argentina \\
LL & Revista Jurídica Argentina La Ley / La ley \\
LLBA & La ley Buenos Aires / La ley \\
TySS & Trabajo y Seguridad Social / La ley \\
TP & Trámite parlamentario / Cámara de Diputados de la Nación \\
TRIBUNALES & \\
CNCiv. & Cámara Nacional de Apelaciones en lo Civil \\
C1a.Civ. y Com. & Cámara Primera de Apelaciones en lo Civil y Comercial \\
CS & Corte Suprema de Justicia de la Nación \\
CNTrab. & Cámara Nacional de Apelaciones del Trabajo \\
TTrab & Tribunal del Trabajo \\
\hline
\end{tabular}

Nota:Las referencias bibliográficas correspondientes al trabajo de investigación constituyen el contenido mismo de la presente bibliografía. 